\title{
A Class of Generalized Differential Operators with Infinitely Many Discontinuous Points
}

\author{
Yurong Li, Jiong Sun, and Siqin Yao \\ School of Mathematical Sciences, Inner Mongolia University, Hohhot 010021, China \\ Correspondence should be addressed to Jiong Sun; masun@imu.edu.cn
}

Received 23 March 2014; Accepted 27 May 2014; Published 15 June 2014

Academic Editor: Valery Serov

Copyright (C) 2014 Yurong Li et al. This is an open access article distributed under the Creative Commons Attribution License, which permits unrestricted use, distribution, and reproduction in any medium, provided the original work is properly cited.

We define a class of differential operators $T$ which have infinitely many discontinuous points, and investigate the kernel of $T$ in terms of operator theory. It is shown that the solutions of $T$ have exponential behavior, and the dimension of the kernel is given.

\section{Introduction and Motivations}

In the study of ergodicity of billiard flows, a very important question concerns the hyperbolicity of these flows $[1,2]$. Hyperbolicity is defined in the language of the linearized system; this is the situation where trajectories close to a given trajectory either diverge exponentially in time from it or converge in the same way. The differential operator $T$ we study is associated with the linearization of the flow; hence we ask whether solutions to $T f=0$ have the corresponding property. However, this is a paper not about ergodic theory, but about the spectral theory $[3,4]$ of a class of generalized differential operators which comes up naturally in this context.

The first class of chaotic billiards was introduced by Sinai in [5]; he proved the ergodicity of plenary dispersing billiards. It took more than 30 years; until 2003, Bálint et al. were able to prove ergodicity for multidimensional dispersing billiard in [6]. However it remains an important and difficult question to study hyperbolicity for nondispersing chaotic billiards, as well as high dimensional Bunimovich type billiards. We plan to give a self-contained approach using spectral theory to study the asymptotic behavior of functions in the kernels of a class of ordinary differential operators, motivated by asymptotic behavior of Jacobian filed of certain dynamical systems with singularities, especially billiards.

In this paper we construct a new class of generalized differential operators associated with the impulsive equations.
The differential operators we deal with are second order, matrix coefficient Schrödinger operators with infinitely many discontinuous points. These kinds of operators are more general than those occurring in billiard flow, but include these as a special case. In this case, the jump conditions correspond to the reflections. We investigate the exponential behavior of functions in the null-space of $T$ in terms of operator theory. On this basis, we obtain the relation between the minimal and maximal operators associated with the weighted operators and then characterize the dimension of the kernel. The results of this paper extend the result in the papers of Kauffman and Zhang [7] and Zhang and Lian [8] to more general case, which gives some hope that the structure of the differential operators may be used later to analyze some of the problems of greater interest in multidimensional billiards.

This paper is divided into five sections: the first section introduces the research background; the second section describes the main results derived in this paper; they are formulated in Theorems 7 and 8; in the third section, we studied the differential operator $T$; then, in the last two sections, we give the proof of the two main theorems.

\section{Statement of the Main Results}

Throughout this paper, we will let $0=t_{0}<t_{1}<\cdots<t_{i}<\cdots$ be a partition $\mathscr{P}$ of $[0, \infty), m \geq 1$. Denote $I_{i}=\left[t_{i}, t_{i+1}\right)$, for all $i=0,1,2, \ldots$. Denote $u \cdot v$ as the inner product of 
vectors $u, v \in \mathbb{C}^{m}$. Let $I$ be a real interval; denote by $L^{2}(I)$ the Hilbert space of all measurable functions $f$ from $I$ to $\mathbb{C}^{m}$, such that

$$
\int_{I}|f|^{2} d t<\infty
$$

with inner product

$$
[f, g]=\int_{I}(f \cdot g) d t
$$

Definition 1. Let $l$ be the differential expression on an interval $I$ defined by

$$
l f=-f^{\prime \prime}+A(t) f
$$

where $A(t)$ is a positive semidefinite symmetric real $m \times m$ matrix for each $t \in R^{+}$and a continuous function of $t$.

Definition 2. Let $l_{p}$ be a set of all sequences of complex numbers $\left\{\xi_{k}\right\}_{k=1}^{\infty}$, which satisfy the condition $\sum_{k=1}^{\infty}\left|\xi_{k}\right|^{p}<$ $\infty$. In this paper, every element of the sequence is a $q$ dimensional column vector; we denote $l_{p}^{q}$ as a set, which satisfy the following condition: $\xi=\left\{\xi_{k}\right\}_{k=1}^{\infty} \in l_{p}^{q}$; then $\xi_{k}=\left(\xi_{k}^{1}\right.$, $\left.\xi_{k}^{2}, \ldots, \xi_{k}^{q}\right)^{\top}(k=1,2, \ldots)$ and $\left\{\xi_{k}^{j}\right\}_{k=1}^{\infty} \in l_{p}$, for $j=1, \ldots, q$.

Definition 3. Let $F\left([0, \infty), \mathbb{C}^{m}\right)$ denote the set of all measurable functions $f$ from $[0, \infty)$ to $\mathbb{C}^{m}$, such that $f$ is differentiable (in distribution sense) almost everywhere. For any $f \in F\left([0, \infty), \mathbb{C}^{m}\right)$, and the partition $\mathscr{P}$ of $[0, \infty)$, define the operator:

$$
\begin{aligned}
\Lambda: F\left([0, \infty), \mathbb{C}^{m}\right) & \longrightarrow l_{0}^{4 m} \\
f & \longmapsto a,
\end{aligned}
$$

where $a=\left\{a_{i}\right\}_{i=1}^{\infty}$ and $a_{i}=\left(f\left(t_{i}-0\right), f^{\prime}\left(t_{i}-0\right), f\left(t_{i}\right), f^{\prime}\left(t_{i}\right)\right)^{T}$ is a $4 m \times 1$ matrix.

Definition 4. Let

$$
T: D(T) \subset L_{2}\left([0, \infty), \mathbb{C}^{m}\right) \longrightarrow L_{2}\left([0, \infty), \mathbb{C}^{m}\right) \times l_{0}^{2 m}
$$

be a linear operator defined as

$$
\begin{gathered}
T f(t)=(g(t), b), \\
g(t)=l f(t), \quad t \in I_{i}, \quad i=0,1,2, \ldots, \\
b=\widetilde{l} f
\end{gathered}
$$

with domain

$$
\begin{aligned}
D(T)= & \left\{f \in L_{2}\left([0, \infty), \mathbb{C}^{m}\right) \mid\right. \\
& \left.f^{\prime} \in A C\left(I_{i}\right), \sum_{i=0}^{\infty}\|l f\|_{2, I_{i}}^{2}<\infty\right\},
\end{aligned}
$$

where $\widetilde{l}=\left(B\left(t_{i}\right),-I\right) \Lambda$, and $A C\left(I_{i}\right)$ denotes the set of absolutely continuous complex valued functions on $I_{i}$, and, for each $i \in$ $\mathbb{N}$,

$$
B\left(t_{i}\right)=\left(\begin{array}{cc}
U_{i} & 0 \\
O_{i} & U_{i}
\end{array}\right)_{2 m \times 2 m}
$$

and the following holds:

(1) $U_{i}^{*} U_{i}=I$, where $I$ is an identity $m \times m$ matrix;

(2) $U_{i}^{*} O_{i}$ is a symmetric operator on the $\mathbb{R}^{m}$;

(3) there exists a universal constant $C_{1}>0$, such that for any unit vector $u \in \mathbb{R}^{m}$, any $t>0$, and any $i \in \mathbb{N}$,

$$
C_{1} \geq\left(U_{i}^{*} O_{i} u, u\right) \geq 0, \quad\|A(t)\|<C_{1} .
$$

Remark 5. In Definition 4, (8) represents the jump conditions at points $\left\{t_{i}\right\}$. The range of $b$ is in $l_{0}^{2 m}$, as one can check that

$$
b=\widetilde{l} f=\left(B\left(t_{i}\right),-I\right) \Lambda f=B\left(t_{i}\right)\left(\begin{array}{c}
f\left(t_{i}-0\right) \\
f^{\prime}\left(t_{i}-0\right)
\end{array}\right)-\left(\begin{array}{c}
f\left(t_{i}\right) \\
f^{\prime}\left(t_{i}\right)
\end{array}\right) .
$$

Definition $6\left(\Omega_{\epsilon, \delta}\right.$ condition). Let $\epsilon>0, \delta>0 . \Omega_{\epsilon, i}$ be a set of all vectors $v \in \mathbb{R}^{m}$ such that

$$
U_{i}^{*} O_{i} v \cdot v \geq \epsilon|v|^{2} .
$$

For any $f:[0, \infty) \rightarrow \mathbb{R}^{m}$, we say that $f \in \Omega_{\epsilon, \delta}$ if there exists a subsequence $\left\{i_{k}\right\} \subset \mathbb{N}$ with

$$
\lim _{n \rightarrow \infty} \inf \frac{1}{n} \sum_{k \leq n}\left(t_{i_{k}}-t_{i_{k}-1}\right) \geq \delta
$$

such that $f\left(t_{i_{k}}-0\right) \in \Omega_{\epsilon, i_{k}}$ for all $i_{k}, k \in \mathbb{N}$.

Note that any function $f \in \Omega_{\epsilon, \delta}$ has a certain nice property that

$$
U_{i_{k}}^{*} O_{i_{k}}|f|^{2}\left(t_{i_{k}}-0\right) \geq \epsilon\left|f\left(t_{i_{k}}-0\right)\right|^{2}
$$

for a subsequence $t_{i_{k}}$ which are not too close to each other.

The main results we derived are Theorems 7 and 8 .

Theorem 7. Let $f \in \operatorname{ker}(T)$, and there exist $\delta>0, \epsilon>0$ such that $f \in \Omega_{\epsilon, \delta}$ for interval I. Then one has the following.

(1) If $|f|$ is nonincreasing, then there exists $\alpha_{1}>0$, such that, for all $\lambda \in\left(0, \alpha_{1}\right)$ and $t \in I$,

$$
|f(t)| \leq e^{-\lambda t}|f(0)|
$$

(2) If $|f|$ is nondecreasing, then there exists $\alpha_{2}>0$, such that, for all $\lambda \in\left(0, \alpha_{2}\right)$ and $t \in I$,

$$
|f(t)| \geq e^{\lambda t}|f(0)|
$$

Next theorem characterizes the dimension of the kernel of $T$. 
Theorem 8. There exist exactly $m$ linearly independent bounded solutions to the equation $\mathrm{Tf}=0$.

Example 9. Consider the one-dimensional case; that is, $m=$ 1. Let $U_{i}=1, O_{i}$ be a real number, and let $A(t)=a(t)$ be a continuous function of $t$ and satisfy the following conditions: $a(t) \geq 0, \exists C_{1}>0$, s.t., $\|a(t)\|<C_{1}$; then the equation $T f=0$ becomes

$$
\begin{gathered}
f^{\prime \prime}(t)=a(t) f \\
f\left(t_{i}-0\right)-f\left(t_{i}\right)=0, \\
f^{\prime}\left(t_{i}-0\right)+O_{i} f\left(t_{i}-0\right)-f^{\prime}\left(t_{i}\right)=0 .
\end{gathered}
$$

It describes the movement of the billiard particle in a smooth table; when it reaches the border, its position is not changed, but the direction is changed. Theorems 7 and 8 hold for this special equation; that is, the solutions of (18) have exponential behavior, and there exists exactly one linearly independent bounded solution.

\section{Characterization of the Generalized Differential Operator $T$}

In this section, we give a characterization on the generalized differential operator $T$.

Definition 10. Define an operator $W: D(W) \subset l_{2}^{2 m} \rightarrow l_{2}^{2 m}$, such that

$$
\begin{gathered}
W(a)=\widehat{a}, \quad \forall a \in l_{2}^{2 m}, \\
\widehat{a_{i}}=Q_{i} a_{i}-X_{i} a_{i+1},
\end{gathered}
$$

where, for $i=1,2, \ldots, Q_{i}$ and $X_{i}$ are $2 m \times 2 m$ nonsingular matrices with $\left\|Q_{i}\right\|_{\infty} \leq C,\left\|X_{i}\right\|_{\infty} \leq C$ for some $C>0$, with

$$
D(W)=\left\{a \in l_{2}^{2 m} \mid W(a) \in l_{2}^{2 m}\right\} .
$$

Lemma 11. $W$ is surjective.

Proof. $W$ can be written as

$$
W=\left(\begin{array}{ccccc}
Q_{1} & -X_{1} & 0 & 0 & \cdots \\
0 & Q_{2} & -X_{2} & 0 & \cdots \\
0 & 0 & Q_{3} & -X_{3} & \cdots \\
\vdots & \vdots & \vdots & \vdots & \ddots
\end{array}\right)
$$

Since $Q_{i}$ and $X_{i}$ are nonsingular matrices, so $W$ is an invertible matrix. For any $\widehat{a} \in l_{2}^{2 m}$, let $a=W^{-1} \widehat{a}$. Then we can check that $W(a)=\widehat{a}$ and $a \in l_{2}^{2 m}$.

In order to study the operator $T$, we first introduce an operator $L$ which was studied in $[7,9]$ and review some properties of $L$.

Definition 12. Let $L$ be a differential operator defined by

$$
L f(t)=l f(t), \quad \forall t \in I_{i} \cap I, i=0,1,2, \ldots,
$$

satisfying the following boundary conditions: for any $i \in \mathbb{N}$,

$$
\left(f\left(t_{i}\right), f^{\prime}\left(t_{i}\right)\right)^{T}=B\left(t_{i}\right)\left(f\left(t_{i}-0\right), f^{\prime}\left(t_{i}-0\right)\right)^{T},
$$

where $U_{i}, O_{i}$ are the same as in the definition of $T$.

Lemma 13 (see [7]). Let $L_{M}$ be the maximal operator of $L$, and let $L_{0}$ be the minimal operator. Then the adjoint operator of $L_{0}$ satisfies

$$
L_{0}^{*}=L_{M} .
$$

Lemma 14 (see [7]). For all $f$ in the domain of $L_{0}$,

$$
[L f, f] \geq \eta\|f\|^{2}
$$

where $\eta$ is a universal positive constant.

Lemma 15 (see [9]). Let $B$ be a densely defined closed operator on a Hilbert space. Denote the range of $B$ by $\mathscr{R}(B)$ and the nullspace of $B$ by $\mathcal{N}(B)$. Then

$$
\mathscr{R}\left(B^{*}\right)^{\perp}=\mathcal{N}(B) .
$$

Based on all the above properties for operator $L$, we obtain the following result.

Lemma 16. Let $g \in L_{2}\left([0, \infty), \mathbb{C}^{m}\right)$. Then there exists $f \in$ $D(T)$ such that

$$
T f(t)=(g, 0) .
$$

Proof. By Lemma 14, we know that $\mathcal{N}\left(L_{0}\right)=\{0\}$. From Lemmas 13 and 15, it is easy to show that $\mathscr{R}\left(L_{M}\right)^{\perp}=\mathscr{N}\left(L_{0}\right)$. So $L_{M}$ is surjective. Then we have, for all $g \in L_{2}\left([0, \infty), \mathbb{C}^{m}\right)$, $\exists f \in D\left(L_{M}\right)$, such that $L_{M} f=g$. But by the definition of $L$, we know that $T f=\left(L_{M} f, 0\right)$, which gives the result.

Lemma 17. Assuming that there exists $d>0$, such that

$$
t_{i+1}-t_{i} \leq d, \quad \forall i \in \mathbb{N},
$$

then the range of $T$ is a subset of $\mathscr{H}:=L_{2}\left([0, \infty), \mathbb{C}^{m}\right) \times l_{2}^{2 m}$.

Proof. Since $f \in L_{2}\left([0, \infty), \mathbb{C}^{m}\right)$ and $l f \in L_{2}\left([0, \infty), \mathbb{C}^{m}\right)$, so

$$
\begin{aligned}
& \left(\|f\|_{2, I_{0}},\|f\|_{2, I_{1}}, \ldots\right) \in l_{1}, \\
& \left(\|l f\|_{2, I_{0}},\|l f\|_{2, I_{1}}, \ldots\right) \in l_{1} .
\end{aligned}
$$

There exists $K>0$, such that, on each $I_{i}$,

$$
\|f\|_{\infty, I_{i}}+\left\|f^{\prime}\right\|_{\infty, I_{i}} \leq K\left(\|f\|_{2, I_{i}}+\|l f\|_{2, I_{i}}\right) \text {. }
$$

Therefore $\left\{f\left(t_{i}-0\right)\right\}_{i=1}^{\infty}, \quad\left\{f\left(t_{i}-0\right)^{\prime}\right\}_{i=1}^{\infty}, \quad\left\{f\left(t_{i}\right)\right\}_{i=0}^{\infty}$, and $\left\{f\left(t_{i}^{\prime}\right)\right\}_{i=0}^{\infty}$ are all in $l_{2}^{m}$. It follows that

$$
\begin{gathered}
\left\{U_{i} f\left(t_{i}-0\right)-f\left(t_{i}\right)\right\}_{i=1}^{\infty} \in l_{2}^{m}, \\
\left\{U_{i} f^{\prime}\left(t_{i}-0\right)+O_{i} f\left(t_{i}-0\right)-f^{\prime}\left(t_{i}\right)\right\}_{i=1}^{\infty} \in l_{2}^{m} .
\end{gathered}
$$

Thus the range of $T$ is contained in $L_{2}\left([0, \infty), \mathbb{C}^{m}\right) \times l_{2}^{2 m}$. 
Next we recall Gronwall's inequality, which will be used in our proof that $T$ is subjective below.

Lemma 18 (Gronwall's inequality). Let $f(t)>0$ be continuous on $[a, b]$ and satisfy

$$
f(t) \leq \alpha+\int_{a}^{t} \beta(s) f(s) d s
$$

where $\alpha>0$ and $\beta>0$ is continuous on $[a, b]$. Then

$$
f(t) \leq \alpha \exp \int_{a}^{t} \beta(s) d s
$$

for $t \in[a, b]$.

Proposition 19. $T: L_{2}\left([0, \infty), \mathbb{C}^{m}\right) \rightarrow \mathscr{H}$ is surjective.

Proof. Let $(g, x, y) \in \mathscr{H}$. By Lemma 16, there exists $f \in D(T)$, such that

$$
\operatorname{Tf}(t)=(g, 0,0) .
$$

We just need to find a $h \in D(T)$, such that $T h=(0, x, y)$. By Definition 4, that is,

$$
\begin{gathered}
h^{\prime \prime}(t)=A(t) h(t) t \in I_{i}, \quad i=0,1,2, \ldots, \\
x_{i}=U_{i} h\left(t_{i}-0\right)-h\left(t_{i}\right), \quad i \in \mathbb{N}, \\
y_{i}=U_{i} h^{\prime}\left(t_{i}-0\right)+O_{i} h\left(t_{i}-0\right)-h^{\prime}\left(t_{i}\right), \quad i=0,1,2, \ldots
\end{gathered}
$$

For each $i \in \mathbb{N}$, let $F_{i}(t)$ be the fundamental solution matrix of

$$
\frac{d}{d t}\left(\begin{array}{c}
h(t) \\
h^{\prime}(t)
\end{array}\right)=\left(\begin{array}{cc}
0 & I \\
A(t) & 0
\end{array}\right)\left(\begin{array}{c}
h(t) \\
h^{\prime}(t)
\end{array}\right), \quad t \in I_{i}
$$

with $F_{i}\left(t_{i}\right)=I$. Since we assumed that $\left|I_{i}\right| \leq d$, for all $i \in \mathbb{N}$, so there exists a uniform constant $C_{d}$, such that $\left\|F_{i}(t)\right\|_{\infty} \leq C_{d}$. By Lemma 11, we choose $Q_{i}=\left(\begin{array}{cc}U_{i} & 0 \\ O_{i} & U_{i}\end{array}\right)$, and $X_{i}^{-1}=F_{i}\left(t_{i+1}-0\right)$, then we know that there exist $a, b \in l_{2}^{m}$, such that

$$
W(a, b)^{T}=(x, y)^{T}
$$

That is,

$$
\left(\begin{array}{c}
x_{i} \\
y_{i}
\end{array}\right)=Q_{i}\left(\begin{array}{c}
a_{i} \\
b_{i}
\end{array}\right)-X_{i}\left(\begin{array}{c}
a_{i+1} \\
b_{i+1}
\end{array}\right)
$$

Letting $h:[0, \infty) \rightarrow \mathbb{C}^{m}$ satisfy $h^{\prime} \in A C\left(I_{i}\right)$ and

$$
h\left(t_{i}-0\right)=a_{i}, \quad h^{\prime}\left(t_{i}-0\right)=b_{i},
$$

then we have

$$
\left(\begin{array}{l}
x_{i} \\
y_{i}
\end{array}\right)=Q_{i}\left(\begin{array}{c}
h\left(t_{i}-0\right) \\
h^{\prime}\left(t_{i}-0\right)
\end{array}\right)-X_{i}\left(\begin{array}{c}
h\left(t_{i+1}-0\right) \\
h^{\prime}\left(t_{i+1}-0\right)
\end{array}\right)
$$

Let

$$
\begin{gathered}
h\left(t_{i}\right)=U_{i} h\left(t_{i}-0\right)-x_{i}, \\
h^{\prime}\left(t_{i}\right)=U_{i} h^{\prime}\left(t_{i}-0\right)+O_{i} h\left(t_{i}-0\right)-y_{i},
\end{gathered}
$$

and then we get

$$
\begin{gathered}
x_{i}=U_{i} h\left(t_{i}-0\right)-h\left(t_{i}\right), \\
y_{i}=U_{i} h^{\prime}\left(t_{i}-0\right)+O_{i} h\left(t_{i}-0\right)-h^{\prime}\left(t_{i}\right), \\
\left(\begin{array}{c}
h\left(t_{i+1}-0\right) \\
h^{\prime}\left(t_{i+1}-0\right)
\end{array}\right)=X_{i}^{-1}\left(\begin{array}{c}
h\left(t_{i}\right) \\
h^{\prime}\left(t_{i}\right)
\end{array}\right) \\
=F_{i}\left(t_{i+1}-0\right)\left(\begin{array}{c}
h\left(t_{i}\right) \\
h^{\prime}\left(t_{i}\right)
\end{array}\right) .
\end{gathered}
$$

It follows that $T h=(0, x, y)$.

In the following, it is enough to show that $h \in$ $L_{2}\left([0, \infty), \mathbb{C}^{m}\right)$. On each $I_{i}$, we have

$$
\frac{d}{d t}\left(\begin{array}{c}
h(t) \\
h^{\prime}(t)
\end{array}\right)=\left(\begin{array}{cc}
0 & I \\
A(t) & 0
\end{array}\right)\left(\begin{array}{c}
h(t) \\
h^{\prime}(t)
\end{array}\right)
$$

Denote

$$
B(t)=\left(\begin{array}{cc}
0 & I \\
A(t) & 0
\end{array}\right), \quad X(t)=\left(\begin{array}{c}
h(t) \\
h^{\prime}(t)
\end{array}\right)
$$

Then

$$
X^{\prime}(t)=B(t) X(t) .
$$

By hypothesis of $A(t)$, we know that $\exists K$, such that $\|B(t)\|_{\infty} \leq$ $K$ and $\left|I_{i}\right| \leq d$; thus on each $I_{i}$

$$
\begin{gathered}
\int_{t_{i}}^{t} X^{\prime}(s) d s=\int_{t_{i}}^{t} B(s) X(s) d s=X(t)-X\left(t_{i}\right), \\
\|X(t)\|_{\infty, I_{i}} \leq\left\|X\left(t_{i}\right)\right\|_{\infty}+\int_{t_{i}}^{t}\|B(s)\|_{\infty}\|X(s)\|_{\infty} d s .
\end{gathered}
$$

By Gronwall's inequality,

$$
\|X(t)\|_{\infty, I_{i}} \leq\left\|X\left(t_{i}\right)\right\|_{\infty} \exp \int_{t_{i}}^{t}\|B(s)\|_{\infty} d s \leq\left\|X\left(t_{i}\right)\right\|_{\infty} e^{d K} .
$$

Combining all these facts together, we get

$$
\|X(t)\|_{2, I_{i}} \leq \sqrt{d m}\left\|X\left(t_{i}\right)\right\|_{\infty} e^{d K} .
$$

Therefore

$$
\begin{aligned}
\|h\|_{2,[0, \infty)}^{2} & \leq\|X(t)\|_{2,[0, \infty)}^{2} \leq \sum_{i=0}^{\infty}\|X(t)\|_{2, I_{i}}^{2} \\
& \leq d m e^{2 d K} \sum_{i=0}^{\infty}\left\|X\left(t_{i}\right)\right\|_{\infty}^{2} \\
& \leq d m e^{2 d K} \sum_{i=0}^{\infty}\left(\left|h\left(t_{i}\right)\right|^{2}+\left|h^{\prime}\left(t_{i}\right)\right|^{2}\right)<\infty .
\end{aligned}
$$

Thus $f+h \in D(T)$ satisfies

$$
T(f+h)(t)=(g(t), x, y) .
$$

This completes the proof of Proposition 19. 


\section{Exponential Behavior of Solutions: Proof of Theorem 7}

In this section, we give the proof of Theorem 7 by considering the exponential behavior of the null space of $T$.

For any $f \in \operatorname{ker}(T)$, we denote

$$
k_{f}(t)=\frac{|f|^{\prime}}{|f|}=\frac{f^{\prime} \cdot f}{f \cdot f} .
$$

Note that $|f|=\sqrt{(f \cdot f)}$, so

$$
|f|^{\prime}=(\sqrt{(f \cdot f)})^{\prime}=\frac{(f \cdot f)^{\prime}}{2 \sqrt{f \cdot f}}=\frac{2 f^{\prime} \cdot f}{2 \sqrt{f \cdot f}}=\frac{f^{\prime} \cdot f}{\sqrt{f \cdot f}} .
$$

Lemma 20. Let $f \in \Omega_{\epsilon, \delta}$ for interval I. Then there exists a constant $r>0$, and $\lambda=\lambda_{\epsilon, \delta}>0$, such that for any $t>r$

$$
\frac{1}{t} \int_{[0, t]}\left|k_{f}(s)\right| d s>\lambda .
$$

Proof. Assume that there exists $\left\{i_{k}\right\} \subset \mathbb{N}$, such that

$$
\lim _{n \rightarrow \infty} \inf \frac{1}{n} \sum_{k \leq n}\left(t_{i_{k}}-t_{i_{k}-1}\right) \geq \delta,
$$

since $f \in \operatorname{ker}(T)$ and $f \in \Omega_{\epsilon, \delta}$, so

$$
\begin{aligned}
& \left(f^{\prime} \cdot f\right)\left(t_{i_{k}}\right)-\left(f^{\prime} \cdot f\right)\left(t_{i_{k}}-0\right) \\
& \quad=U_{i_{k}} O_{i_{k}}|f|^{2}\left(t_{i_{k}}-0\right) \geq \epsilon|f|^{2}\left(t_{i_{k}}-0\right) .
\end{aligned}
$$

This implies that

$$
\left|k_{f}\left(t_{i_{k}}\right)-k_{f}\left(t_{i_{k}}-0\right)\right|=\frac{U_{i_{k}} O_{i_{k}}|f|^{2}\left(t_{i_{k}}-0\right)}{f \cdot f} \geq \epsilon .
$$

Thus

$$
\frac{1}{t} \int_{[0, t]}\left|k_{f}(s)\right| d s>\frac{1}{t} \sum_{t_{i_{k}}<t}\left(k_{f}\left(t_{i_{k}}\right)-k_{f}\left(t_{i_{k}}-0\right)\right)>\frac{\epsilon}{t} b_{t},
$$

where $b_{t}$ is the number of $t_{i_{k}}$, such that $t_{i_{k}}<t$. But we know that

$$
\lim _{t \rightarrow \infty} \inf \frac{b_{t}}{t} \geq \delta
$$

so there exists $r>0$, such that, for any $t>r$,

$$
\frac{1}{t} \int_{[0, t]}\left|k_{f}(s)\right| d s>\lambda,
$$

where $\lambda=\delta \epsilon$.

Now we are ready to prove Theorem 7.
Proof. By the definition of $k_{f}$, we know that

$$
k_{f}(t)=\frac{|f|^{\prime}}{|f|}=(\ln |f|)^{\prime}(t) .
$$

Thus

$$
\begin{gathered}
\ln |f|=\int_{0}^{t} k_{f}(s) d s+c, \\
|f|(t)=c e^{\int_{0}^{t} k_{f}(s) d s}=|f(0)| e^{\int_{0}^{t} k_{f}(s) d s} .
\end{gathered}
$$

(1) Since $|f|$ is nonincreasing, then $k_{f}(t) \leq 0$; by Lemma 20 , we have

$$
\int_{0}^{t} k_{f}(s) d s=-\int_{0}^{t}\left|k_{f}(s)\right| d s<-\lambda t,
$$

so

$$
|f(t)| \leq e^{-\lambda t}|f(0)| .
$$

(2) Since $|f|$ is nondecreasing, then $k_{f}(t) \geq 0$; by Lemma 20 , we have

$$
\int_{0}^{t} k_{f}(s) d s=\int_{0}^{t}\left|k_{f}(s)\right| d s>\lambda t,
$$

so

$$
|f(t)| \geq e^{\lambda t}|f(0)| .
$$

\section{Proof of Theorem 8}

In this section, we give the proof of Theorem 8 . First we obtain the relation between the minimal and maximal operators associated with the weighted operators $M_{I}$ and then characterize the dimension of the kernel of $T$.

Definition 21. Let $L$ be defined as Definition 12, $\langle t\rangle=\sqrt{t^{2}+1}$. Define

$$
M_{I}=\langle t\rangle L\langle t\rangle, \quad \forall t \in I_{i} \cap I, i=0,1,2, \ldots .
$$

Definition 22. Let the maximal operator $M_{M, I}$ associated with $M_{I}$ be the restriction of $M_{I}$ on $D_{M}$, with

$$
\begin{gathered}
D_{M}=\left\{f \in L^{2}(I) \cap D(L) \mid M_{I}(f) \in L^{2}(I),\right. \\
\left.f^{\prime} \in A C\left(I_{i} \cap I\right), \forall i=0,1,2, \ldots\right\} .
\end{gathered}
$$

Let $\widehat{D_{I}}$ be the set of all $f \in D_{M}$, such that $f \in C^{\infty}\left(I_{i} \cap I\right)$, for all $i \in \mathbb{N}$ and $f$ vanishes in a neighborhood of both "end points" of $I$ and $\operatorname{supp}(f)$ is compact. Denote $\widehat{M_{I}}$ to be $M_{I}$ restricting on $\widehat{D_{I}}$; then we define the minimal operator $M_{0, I}$ to be the smallest closed operator in $L^{2}(I)$ which extends $\widehat{M}_{I}$ and denote by $D_{0}$ the domain of $M_{0, I}$. 
Lemma 23. If $M_{I}$ is defined as in Definition 21 on $I$, then

$$
M_{0, I} \subseteq M_{M, I},
$$

and, for any $f$ in the domain of $M_{0, I}$, we have $f(0)=0, f^{\prime}(0)=$ 0 . If $I$ is compact, say $I=[0, \mathscr{T}] \subset[0, \infty)$, then for any $f \in D_{0}$, also $f(\mathscr{T})=f^{\prime}(\mathscr{T})=0$.

Proof. Let $\left\{f_{n}\right\}$ be a Cauchy sequence of functions from $\widehat{D_{I}}$ converging to $f$ with $M_{0, I} f_{n}$ converging to $M_{0, I} f$ in $L^{2}(I)$. Then on any compact interval $[\alpha, \beta] \subseteq I_{i}, t \geq 0, f_{n}$ and $f_{n}^{\prime}$ both converge uniformly; therefore $f_{n}^{\prime \prime}$ also converges in each $L^{2}\left(I_{i}\right)$, so $f_{n}^{\prime \prime}$ converges in $L^{2}(I)$. It follows that $f^{\prime}$ is absolutely continuous on each $I_{i}$, and $f^{\prime}(0)=0$, if $I=[0, \mathscr{T}]$; then also $f^{\prime}(\mathscr{T})=0$. Since $f_{n}^{(i)}$ converges to $f^{(i)}$ for $i=0,1,2$, we see that $M_{0, I} f=M_{I} f, M_{I} f$ is in $L^{2}$, and so $f \in D_{M, I}$.

Definition 24 (see [10]). A densely defined operator $S$ on a Hilbert space is said to be symmetric if $[S f, g]=[f, S g]$ for all $f, g$ in the domain of $S$.

Lemma 25. $M_{0, I}$ is a symmetric operator, for $I=[0, \mathscr{T}]$ or $I=[0, \infty)$.

Proof. For any $f, g$ in the domain of $M_{0, I}$, by Lemma 23, we know that $f(0)=f^{\prime}(0)=0$ and $g(0)=g^{\prime}(0)=0$. Without loss of generality, we suppose that $f$ and $g$ are real vector valued functions:

$$
\begin{aligned}
{\left[M_{I} f, g\right]-\left[f, M_{I} g\right] } & \\
= & -\left[\langle t\rangle(\langle t\rangle f)^{\prime \prime}, g\right]+[\langle t\rangle A\langle t\rangle f, g] \\
& +\left[f,\langle t\rangle(\langle t\rangle g)^{\prime \prime}\right]-[f,\langle t\rangle A\langle t\rangle g] \\
= & -\int_{I}\left(\langle t\rangle(\langle t\rangle f)^{\prime \prime} \cdot g-f \cdot\langle t\rangle(\langle t\rangle g)^{\prime \prime}\right)(t) d t \\
& +\left[\langle t\rangle^{2} A f, g\right]-\left[f,\langle t\rangle^{2} A g\right] \\
= & -\left.\left[(\langle t\rangle f)^{\prime} \cdot\langle t\rangle g-\langle t\rangle f \cdot(\langle t\rangle g)^{\prime}\right]\right|_{I} \\
= & -\left.\left[\langle t\rangle^{2} f^{\prime} \cdot g-\langle t\rangle^{2} f \cdot g^{\prime}\right]\right|_{I} .
\end{aligned}
$$

If $I$ is a compact interval $[0, \mathscr{T}]$, then by the fact that

$$
f(\mathscr{T})=f^{\prime}(\mathscr{T})=g(\mathscr{T})=g^{\prime}(\mathscr{T})=0,
$$

we get $\left[M_{I} f, g\right]=\left[f, M_{I} g\right]$.

If $I=[0, \infty)$, by hypotheses on the boundary conditions and $U_{i}^{*} U_{i}=I$, we have

$$
\begin{aligned}
{\left[f^{\prime} \cdot g-f \cdot g^{\prime}\right]\left(t_{i}\right) } \\
=\left[O_{i} f\left(t_{i}-0\right)+U_{i} f^{\prime}\left(t_{i}-0\right)\right] \\
\cdot U_{i} g\left(t_{i}-0\right)-U_{i} f\left(t_{i}-0\right) \\
\cdot\left[O_{i} g\left(t_{i}-0\right)+U_{i} g^{\prime}\left(t_{i}-0\right)\right] \\
=\left[f^{\prime} \cdot g-f \cdot g^{\prime}\right]\left(t_{i}-0\right) .
\end{aligned}
$$

Thus we get

$$
\begin{aligned}
& {\left[M_{I} f, g\right]-\left[f, M_{I} g\right]} \\
& \quad=-\sum_{i=1}^{\infty}\langle t\rangle^{2}\left\{\left[f^{\prime} \cdot g-f \cdot g^{\prime}\right]\left(t_{i}\right)-\left[f^{\prime} \cdot g-f \cdot g^{\prime}\right]\left(t_{i}-0\right)\right\} \\
& \quad=0 .
\end{aligned}
$$

That is, $\left[M_{I} f, g\right]=\left[f, M_{I} g\right]$. So by Definition $24, M_{0, I}$ is symmetric.

Lemma 26. For $h \in L^{2}(I)$, the equation $M_{I} f=h$ has $a$ solution $f$ in the domain of $M_{0, I}$ if and only if $h$ is orthogonal to all solutions of $M_{M, I} g=0$.

Proof. Assume that, for $h \in L^{2}(I)$, the equation $M_{I} f=h$ has a solution $f \in D_{0}$. For any $g \in D_{M}$, such that $M_{I} g=0$, we have

$$
[h, g]=\left[M_{0, I} f, g\right]=\left[f, M_{I} g\right]=0 .
$$

Conversely, if $h$ is orthogonal to all solutions of $M_{M, I} g=0$, choose $f$ such that $M_{I} f=h$ and $f(\alpha)=f^{\prime}(\alpha)=0$. We need to show that $f(\beta)=f^{\prime}(\beta)=0$. Using the same calculation as in the proof of Lemma 25, we get

$$
\left[M_{I} f, g\right]-\left[f, M_{I} g\right]=f(\beta) \cdot g^{\prime}(\beta)-f^{\prime}(\beta) \cdot g(\beta) \text {. }
$$

On the other hand, Consider

$$
\left[M_{I} f, g\right]-\left[f, M_{M, I} g\right]=\left[M_{I} f, g\right]=[h, g]=0 \text {. }
$$

Thus we get that $f(\beta) \cdot g^{\prime}(\beta)-f^{\prime}(\beta) \cdot g(\beta)=0$ for any $g$ satisfying $M_{I} g=0$. Choose $g \in D_{M}$, s.t. $g(\beta) \neq 0$, but $g^{\prime}(\beta)=$ 0 ; then we get $f^{\prime}(\beta)=0$; similarly, we can get $f(\beta)=0$.

Corollary 27. $I=[\alpha, \beta] \subset[0, \infty)$; letting $\tau$ denote the restriction of $M_{I}$ to domain

$$
\left\{f \in D_{M}(I): f(\alpha)=f^{\prime}(\alpha)=0, f(\beta)=f^{\prime}(\beta)=0\right\},
$$

then $\tau=M_{0, I}$.

Based on all the above results, we can derive the relation between the operators $M_{0, I}$ and $M_{M, I}$.

Proposition 28. If $M_{I}$ is defined as in Definition 21 and $I=$ $[\alpha, \beta] \subset[0, \infty)$, then

$$
M_{0, I}^{*}=M_{M, I}
$$

Proof. Since $M_{0, I}$ is the smallest closed extension of $\widehat{M_{I}}$, it follows that

$$
M_{0, I}^{*}=\widehat{M_{I}^{*}}
$$


As for any $f \in \widehat{D_{I}}$ (then $\left.f \in D_{0}\right)$ and $g \in D_{M, I}$, $\left[\widehat{M_{I}} f, g\right]=$ $\left[f, M_{M, I} g\right]$, then

$$
M_{M, I} \subseteq \widehat{M_{I}^{*}}=M_{0, I}^{*}
$$

Let $f$ be in the domain of $M_{0, I}^{*}$. Since $M_{M, I}$ is surjective for compact $I$, so there is a function $g$ in $D_{M, I}$ such that $M_{M, I} g=$ $M_{0, I}^{*} f ;$ thus

$$
M_{0, I}^{*}(f-g)=0 .
$$

So if $u$ is in the range of $M_{0, I}$ with $M_{0, I} v=u$, we have

$$
[f-g, u]=\left[f-g, M_{0, I} v\right]=\left[M_{0, I}^{*}(f-g), v\right]=0 .
$$

Therefore $f-g$ is orthogonal to the range of $M_{0, I}$, but, by Lemma $26, f-g$ is in the null space of $M_{M, I}$. Hence $f \in D_{M, I}$; that is, $M_{0, I}^{*} \subset M_{M, I}$.

Proposition 29. If $M_{I}$ is defined as in Definition 21 and $I \subset$ $[0, \infty)$, then

$$
M_{0, I}^{*}=M_{M, I}
$$

Proof. By Proposition 28, we only need to show $M_{0, I}^{*} \subset M_{M, I}$. If $g$ is in the domain of $M_{0, I}^{*}$, then, on any compact subinterval $[\alpha, \beta]$ of $I$, we have

$$
\left[M_{I} f, g\right]=\left[f, M_{0, I}^{*} g\right], \quad \forall f \in \widehat{D_{I}}
$$

Thus the restriction of $g$ to $[\alpha, \beta]$ is in the domain of $M_{0, I}^{*}$. But, by Proposition 28, the restriction of $M_{0, I}^{*} g$ to $[\alpha, \beta]$ must agree with $M_{M, I} g$. Since $[\alpha, \beta]$ is arbitrary, the proposition is proved.

We can show that $M_{0, I}$ is semibounded in the next lemma.

Lemma 30. If $M_{I}$ is defined as in Definition 21, for all $f \in D_{0}$,

$$
\left[M_{I} f, f\right] \geq \frac{1}{4}\|f\|^{2}
$$

Proof. By integration by parts we obtain, for $f \in C_{0}^{\infty}\left(\mathbb{R}^{+}\right)$,

$$
\begin{aligned}
{\left[M_{0, I} f, f\right] } & =[\langle t\rangle L(\langle t\rangle f), f] \\
& =-\left[\langle t\rangle(\langle t\rangle f)^{\prime \prime}, f\right]+[\langle t\rangle A\langle t\rangle f, f] \\
& =\left[(\langle t\rangle f)^{\prime},(\langle t\rangle f)^{\prime}\right]+\left[\langle t\rangle^{2} A f, f\right] \\
& \geq\left[(\langle t\rangle f)^{\prime},(\langle t\rangle f)^{\prime}\right] \\
& \geq \frac{1}{4}\|f\|^{2},
\end{aligned}
$$

where the last inequality follows from Hardy's inequality [11].

In order to prove Theorem 8 , we need to introduce the Friedrichs extension of $M_{0, I}$.
Definition 31. With $M_{I}$ as in Definition 21, let $Q_{I}(f, g)=$ $\left[M_{I} f, g\right]$, for all $f, g \in \widehat{D_{I}}$.

It follows from operator theory [9] that the semibounded symmetric operator $M_{0, I}$ has equal deficiency indices, and therefore, by Von Neumann's theorem, such an operator always has self-adjoint extensions. There is a distinguished extension $H$, called the Friedrichs extension [11], which is obtained from the quadratic form associated with $M_{0, I}$.

Proposition 32. $Q_{I}$ is a closable quadratic form and its closure $\widehat{Q}_{I}$ is the quadratic form of a unique self-adjoint operator $H$ defined by

$$
\begin{aligned}
D\left(H_{I}\right)=\left\{f \in L^{2}(I):\left|Q_{I}(f, g)\right|\right. \\
\left.\leq C_{f}[g, g], \forall g \in \widehat{D_{I}}, \text { where } C_{f} \text { is a constant }\right\}, \\
\quad H_{I} f=M_{M, I} f, \quad \forall f \in D\left(H_{I}\right) .
\end{aligned}
$$

Furthermore,

$$
\left[H_{I} f, f\right] \geq \frac{1}{4}[f, f], \quad \forall f \in D\left(H_{I}\right) .
$$

Proof. This is a form of the definition of the Friedrichs extension: for the semibounded symmetric operator $M_{0, I}$ and the closable quadratic form $Q_{I}$, the restriction of $M_{0, I}^{*}$ to the domain of the closure of $Q_{I}$ is in fact the Friedrichs extension. This form is clearly closable. The last inequality follows from the construction of the Friedrichs extension $H_{I}$, together with Lemma 30.

Next proposition tells us the relation between $\operatorname{ker}(T)$ and $\operatorname{ker}\left(M_{I}\right)$.

Proposition 33. $f \in \operatorname{ker}(T)$ is nonincreasing if and only if $g=f /\langle t\rangle$ is a nonincreasing solution to $M_{I} g=0$.

Proof. Let $f \in \operatorname{ker}(T)$; if $|f|$ is nonincreasing, then $f^{\prime} \cdot f<0$. So by east calculation, we have

$$
(g \cdot g)^{\prime}(t)=2 g^{\prime}(t) \cdot g(t)=2 \frac{f^{\prime} \cdot f\left(1+t^{2}\right)-f^{2} t}{\left(1+t^{2}\right)^{2}}<0,
$$

which implies the result.

Now we are ready to prove Theorem 8 .

Proof. It is sufficient to consider real solutions. Let $f$ be any real solution of $T f=0$; for any $i \in \mathbb{N}$, let $t \in I_{i},(f \cdot f)^{\prime}(t)=$ $2 f^{\prime}(t) \cdot f(t)$. Using the fact that $A(t)$ is positive semidefinite for each $t \geq 0$, we have

$$
(f \cdot f)^{\prime \prime}(t)=2\left|f^{\prime}\right|^{2}(t)+2(A(t) f(t) \cdot f(t)) \geq 0 .
$$

On the other hand, for any $i \in \mathbb{N}$,

$$
\left(f \cdot f^{\prime}\right)\left(t_{i}\right)-\left(f \cdot f^{\prime}\right)\left(t_{i}-0\right)=\left(U_{i}^{*} O_{i} f, f\right)\left(t_{i}-0\right) \geq 0 .
$$


This implies that $(f \cdot f)^{\prime}$ is nondecreasing on $[0, \infty]$. Choosing $f$, such that $f(0)=0, f^{\prime}(0) \neq 0$, then $(f \cdot f)^{\prime}(t)>$ $(f \cdot f)^{\prime}(0)=0$, from $(52) ;|f|$ is nondecreasing. Then, by Theorem 7, we know that those solutions have exponentially increasing amplitude; that is, they are unbounded solutions. Clearly $\operatorname{dim}(\operatorname{ker}(T)) \leq 2 m$. The set $\{f \in \operatorname{ker}(T) \mid f(0)=$ $\left.0, f^{\prime}(0) \neq 0\right\}$ is $m$ dimensional, which means that there are at most $m$ linearly independent bounded solutions for $T f=0$.

Now we prove $\operatorname{dim}\left(\operatorname{ker}\left(M_{M, I}\right)\right) \geq m$, using the properties of Friedrichs extension of $M_{0, I}$. Let $H_{I}$ be the Friedrichs extension of $M_{0, I}$; then, by Proposition $32, H_{I}$ is self-adjoint and positive definite. Also for any $f$ in $D\left(H_{I}\right), f(0)=0$. We choose $m$ compactly supported piecewise smooth functions $f_{1}, f_{2}, \ldots, f_{m}$ on $I$, such that the space spanned by $\left\{f_{k}(0)\right.$ | $k=1, \ldots, m\}$ has dimension $m$. Then $\left\{f_{k} \mid k=1, \ldots, m\right\}$ are linearly independent mod $D\left(H_{I}\right)$. It is clear that $f_{k} \in$ $D_{M}$ for $k=1, \ldots, m$; this forces the Fredholm index to increase by $m$. Since $H_{I}$ is already surjective, this produces an $m$ dimensional nonincreasing solution space to $M_{I} f=0$, so $\operatorname{dim}\left(\operatorname{ker}\left(M_{M, I}\right)\right) \geq m$; by Proposition 33, we know that there are at least $m$ linearly independent bounded solutions for $T f=0$. This completes the proof.

\section{Conflict of Interests}

The authors declare that there is no conflict of interests regarding the publication of this paper.

\section{Acknowledgments}

The authors thank the referee for her/his suggestions which have improved the presentation of the paper. The work of the authors is supported by the National Nature Science Foundation of China (Grant no. 11161030) and the third author is supported by the Program of Higher Level Talents of Inner Mongolia University (SPH-IMU).

\section{References}

[1] S. Kerckhoff, H. Masur, and J. Smillie, "Ergodicity of billiard flows and quadratic differentials," Annals of Mathematics, vol. 124, no. 2, pp. 293-311, 1986.

[2] N. Chernov and R. Markarian, Chaotic Billiards, vol. 127 of Mathematical Surveys and Monographs, American Mathematical Society, Providence, RI, USA, 2006.

[3] J. Weidmann, Spectral Theory of Ordinary Differential Operators, vol. 1258 of Lecture Notes in Mathematics, Springer, Berlin, Germany, 1987.

[4] M. A. Naimark, Linear Differential Operators, Harrap, London, UK, 1968.

[5] G. Ya. Sinai, "Dynamical systems with elastic reflections," Russian Mathematical Surveys, vol. 25, pp. 137-189, 1970.

[6] P. Bálint, N. Chernov, D. Szász, and I. P. Tóth, "Geometry of multi-dimensional dispersing billiards," Asterisque, no. 286, pp. 119-150, 2003.

[7] R. M. Kauffman and H. Zhang, "A class of ordinary differential operators with jump boundary conditions," in Evolution Equations, vol. 234 of Lecture Notes in Pure and Applied Mathematics, pp. 253-274, Dekker, New York, NY, USA, 2003.
[8] H. Zhang and J. Lian, "Hyperbolic behavior of jacobi fields along billiard flows," in Proceedings of the 4th International Conference on Impulsive and Hybrid Dynamical Systems, pp. 1794-1798, 2007.

[9] J. Sun and Z. Wang, Spectral Analysis of Linear Operators, Science Press, Beijing, China, 2005.

[10] Z. Cao, Ordinary Differential Operators, Shanghai Scientific and Technical Publishers, Shanghai, China, 1987.

[11] E. Müller-Pfeiffer, Spectral Theory of Ordinary Differential Operators, Ellis Horwood, Chichester, UK, 1981. 


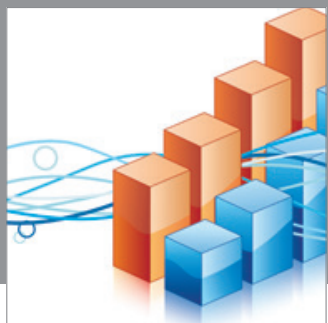

Advances in

Operations Research

mansans

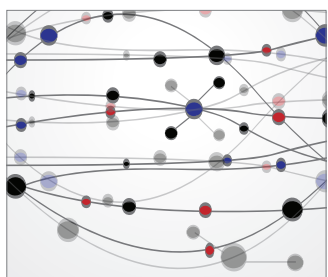

The Scientific World Journal
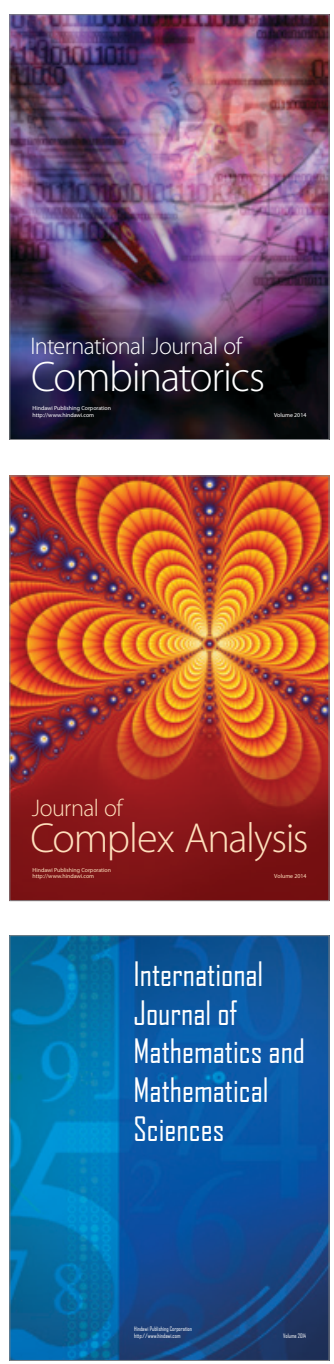
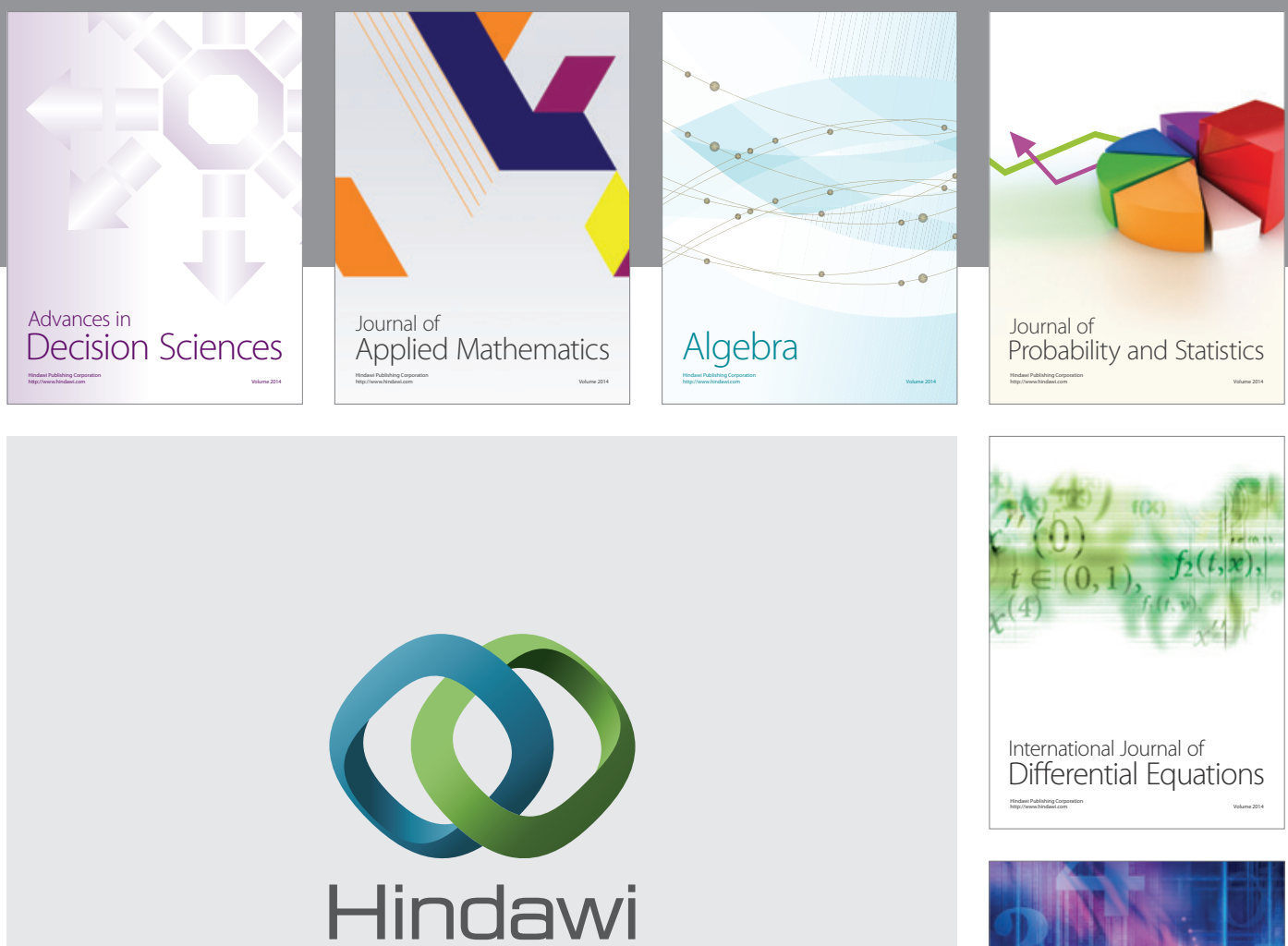

Submit your manuscripts at http://www.hindawi.com
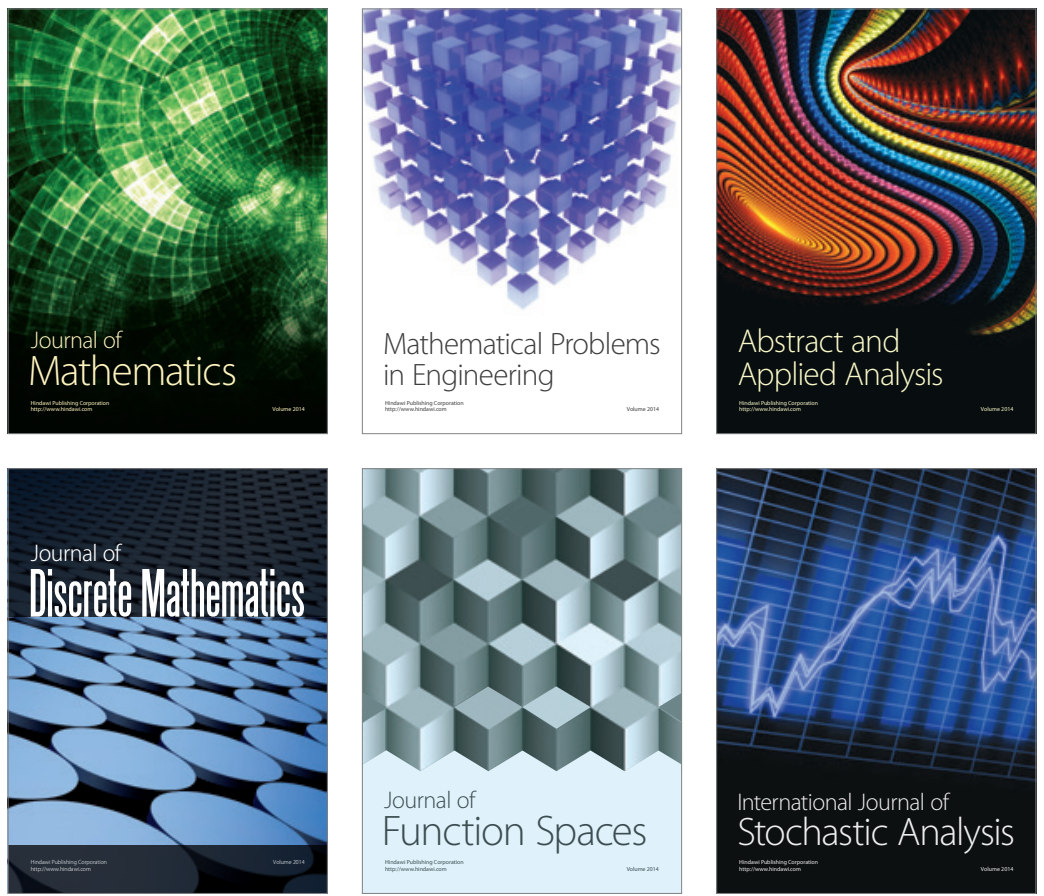

Journal of

Function Spaces

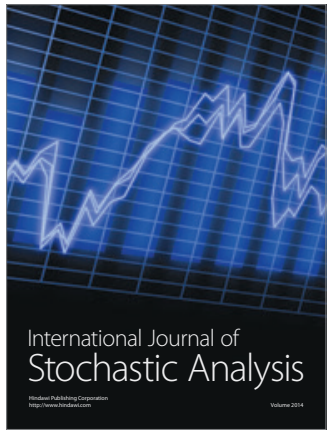

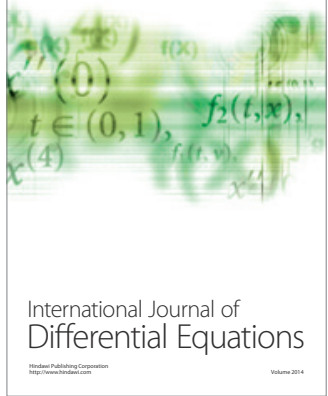
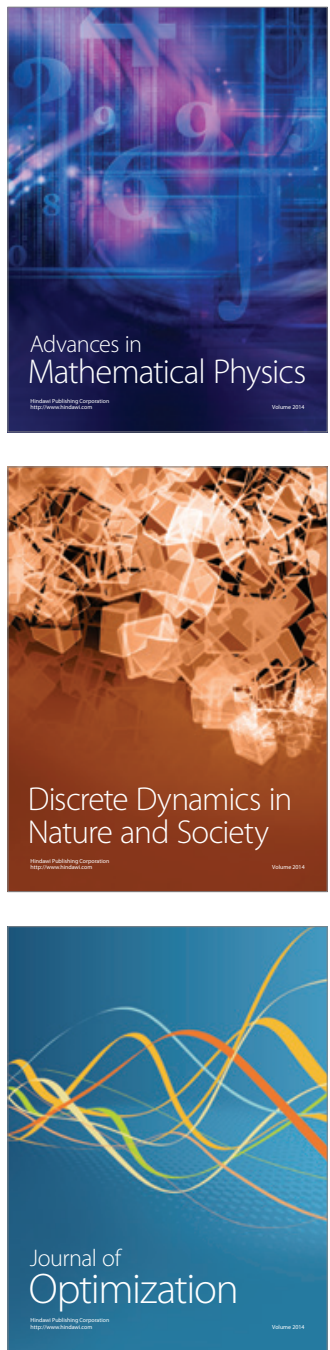